\title{
Prevalence and risk factors of lumbar spondylolisthesis in elderly Chinese men and women
}

\author{
Lai-Chang He ${ }^{1,4}$, Yi-Xiang J Wang ${ }^{1,{ }^{*}}$, Jing-Shan Gong ${ }^{5}$, James F Griffith ${ }^{1}$, Xian-Jun Zeng ${ }^{1,2}$, \\ Anthony WL Kwok ${ }^{1,2}$, Jason CS Leung ${ }^{2}$, Timothy Kwok ${ }^{2,3}$, Anil T Ahuja ${ }^{1}$, and Ping Chung \\ Leung ${ }^{2}$ \\ ${ }^{1}$ Department of Imaging and Interventional Radiology, The Chinese University of Hong Kong, \\ Prince of Wales Hospital, Shatin, New Territories, Hong Kong SAR, China \\ 2Jockey Club Centre for Osteoporosis Care and Control, School of Public Health and Primary \\ Care, The Chinese University of Hong Kong, Prince of Wales Hospital, Shatin, New Territories, \\ Hong Kong SAR, China \\ ${ }^{3}$ Department of Medicine and Therapeutics, The Chinese University of Hong Kong, Prince of \\ Wales Hospital, Shatin, New Territories, Hong Kong SAR, China \\ ${ }^{4}$ Department of Radiology, The First Affiliated Hospital of Nanchang University, Nanchang, China \\ ${ }^{5}$ Department of Radiology, Shenzhen People's Hospital, Jinan University Second Clinical Medical \\ College, Shenzhen, China
}

\section{Abstract}

Objective-A screening survey for osteoporotic fractures in men and women in Hong Kong represents the first large-scale prospective population-based study on bone health in elderly ( $\ 65$ years) Chinese men and women. This study aims to identify the prevalence and potential risk factors of lumbar spondylolisthesis in these subjects.

Methods-The lateral lumbar radiographs of 1,994 male and 1,996 female patients were analysed using the Meyerding classification.

Results-Amongst the men, 380 (19.1\%) had at least one spondylolisthesis and 43 (11.3\%) had slips at two or more levels; 283 had anterolisthesis, 85 had retrolisthesis, whereas 12 subjects had both anterolisthesis and retrolisthesis. Amongst the women, $499(25.0 \%)$ had at least one spondylolisthesis and $69(13.8 \%)$ had slips at two or more levels; 459 had anterolisthesis, 34 had retrolisthesis, whereas 6 subjects had both anterolisthesis and retrolisthesis. Advanced age, short height, higher body mass index (BMI), higher bone mineral density (BMD) and degenerative arthritis are associated with spondylolisthesis. Lower Physical Activity Scale for the Elderly (PASE) score was associated with spondylolisthesis in men; higher body weight, angina and lower grip strength were associated with spondylolisthesis in women.

Conclusion-The male/female ratio of lumbar spondylolisthesis prevalence was 1:1.3 in elderly Chinese. Men are more likely to have retrolisthesis.

\section{Keywords}

Prevalence; Risk factors; Spondylolisthesis; Chinese; Vertebrae

\footnotetext{
*Address correspondence and reprint requests to Dr Yi-Xiang J Wang. Department of Imaging and Interventional Radiology, The Chinese University of Hong Kong, Prince of Wales Hospital, Shatin, New Territories, Hong Kong; yixiang_wang@ @ cuhk.edu.hk Fax (852) 26360012.
} 


\section{Introduction}

Spondylolisthesis is defined as the anterior or posterior migration, or slip, of one vertebra in relation to the next caudal vertebra. It mostly occurs in the lumbar spine and is considered to have two main aetiologies, spondylolytic and degenerative [1]. Spondylolytic spondylolisthesis is distinguished by fracture of the pars interarticularis and is observed primarily during childhood or early adult life [2,3]. Degenerative spondylolisthesis refers to slip without an associated defect in the posterior vertebral ring [4]. Although controversies regarding the pathogenesis of degenerative spondylolisthesis still exist, it is generally acknowledged that degenerative spondylolisthesis is seldom encountered in subjects less than 50 years of age. Degenerative spondylolisthesis is considered a classic example of spinal instability resulting from progressive degeneration of both the facet joints and the intervertebral discs with aging [5].

Although spondylolisthesis is usually asymptomatic, it can progress into spinal stenosis and result in neurogenic symptoms, such as leg pain, numbness, or weakness. Lumbar spondylolisthesis is considered to be a significant source of back pain and disability [6]. Over 300,000 lumbar spine fusions are performed in the USA each year and this number is increasing [7, 8]. Many of these fusions are performed to correct the instability associated with degenerative spondylolisthesis [9-12].

The prevalence of spondylolisthesis has been reported in symptomatic and asymptomatic subjects, and the results vary considerably. Most studies have focused on anatomical features associated with degenerative spondylolisthesis among symptomatic patients. Data from non-clinical, community-based populations are limited. Prevalence estimates among women range from $6 \%$ in Taiwan [13], $8 \%$ in Denmark [14], to 20-25\% in the USA [1517], whereas among men estimates range from $3 \%$ in Taiwan and Denmark to 4-31\% in the USA [13-18]. It has been suggested that women are about three times more likely to be affected by spondylolisthesis than men, and black women are about three times more frequently affected than white women [4, 18-20]. Until now, accurate precise data on spondylolisthesis prevalence among elderly Chinese subjects are lacking.

Using epidemiology data derived from 2,000 Chinese elderly men and 2,000 Chinese elderly women, this study aimed to estimate the prevalence of spondylolisthesis among communitydwelling elderly Chinese subjects, and determine whether certain demographic factors, lifestyle characteristics or medical conditions are associated with the prevalence of this condition. A clearer understanding of the epidemiology of spondylolisthesis is needed to allow a more in-depth patient discussion and to formulate evidence-based treatment plans.

\section{Materials and methods}

Two thousand Chinese men and two thousand Chinese women aged 65 years or above were recruited from the local communities by advertisements placed in housing estates and community centres for older people to take part in a prospective cohort study from August 2001 to March 2003. The recruitment criteria were established so that the study results from the cohort would be applicable to a broad population of similarly aged community-dwelling men and women. The project was designed primarily to examine the bone mineral density (BMD) of older Chinese adults prospectively for 4 years. All subjects were community dwelling, able to walk without assistance, had no bilateral hip replacement and had the potential to survive the duration of a primary study based on their general medical health. No subjects had a known malignancy or previous lumbar spine surgery. Subjects were invited to the research centre for interviews and physical examination. The study protocol was approved by the Chinese University of Hong Kong Ethics Committee. Written informed 
consent was obtained from all subjects. Data from the baseline evaluation were analysed in the current report.

The participants were interviewed using a structured standardised questionnaire. This questionnaire included demographic information, socioeconomic status, medical history related to osteoporosis, history of fracture, current medications (verified by direct inspection or medical record) and alcohol and tobacco consumption. Dietary intake was recorded using a modified Block Food Frequency questionnaire based on the data from the Hong Kong Adult Dietary Survey in 1995 [21]. Physical activity was measured by the Physical Activity Scale for the Elderly (PASE) questionnaire modified for use in older Chinese men and women in Hong Kong [22]. Height and weight of the subjects were measured with indoor clothing but without shoes. Height was measured using the Harpenden stadiometer (Holtain Ltd., Crosswell, UK). Body weight was measured using the physician beam balance scale (Healthometer, Alsip, IL, USA). Both readings were recorded to one decimal place. Grip strength on both hands was measured with a Preston grip dynamometer. Three tests on each hand were performed and the average of the second and third tests on each hand was used. Body mass index (BMI) was calculated using the formula of weight/height ${ }^{2}$ (kilograms per square metre). BMD (grams per square centimetre) at the total hip and spine (L1-L4) was measured by QDR-4,500 W densitometers (Hologic, Inc., Waltham, MA, USA). The in vivo coefficient of variation of the system was 0.9 and $0.7 \%$ at the lumbar spine and at the total hip, respectively.

Left lateral recumbent lumbar spine radiographs were obtained by adjusting exposure parameters according to participants' body weight and height. Only radiographs deemed suitable for accurate assessment of spondylolisthesis were analysed. Spondylolisthesis was defined as a forward slip (anterolisthesis) or backward slip (retrolisthesis) of one vertebral body by at least $5 \%$ in relation to the next most caudal vertebral body and assessed from L1 to S1. Grading of spondylolisthesis was estimated using the Meyerding classification: grade 0, no slip; grade I, $>5 \%$ and $<25 \%$; grade II, 26-50 \%; grade III, 51-75\%; grade IV, 76$100 \%$; and grade V, complete slippage [23]. All lumbar spine radiographs were interpreted and measured by two musculoskeletal radiologists in consensus. This study did not differentiate between spondylolisthesis and spondylolytic spondylolisthesis because only the lateral radiograph was obtained. Anterolisthesis and retrolisthesis are together termed spondylolisthesis. The test-retest reliability assessment conducted on a sample of 100 films indicated $100 \%$ agreement on the presence of spondylolisthesis.

In this study, 24 factors were analysed according to their relationship with spondylolisthesis, including age (years), weight (kilograms), height (metres), BMI (kilograms per square metre), current smoking, current alcohol consumption, hypertension, angina, PASE score, chronic obstructive pulmonary disease (COPD), average left/right grip strength (kilograms), history of fracture, corticosteroid intake, self-reported degenerative arthritis (DA), lumbar spine BMD, hip BMD, low back pain, difficulty bending forward, dietary calcium intake, diabetes and longest occupation involving physical labour; for women number of children, age at menopause and any hormone replacement usage were also analysed. Statistical analyses were performed using the statistical package SAS, version 9.1.3 (SAS Institute, Inc., Cary, NC, USA). Characteristics of participants with and without lumbar spondylolisthesis were compared for the men and women separately. Two sample independent $t$ tests were used for continuous variables, whereas Chi-squared tests were used for categorical variables. Logistic regression was performed for significant factors. All statistical tests were two-sided. An a level of $5 \%$ was used as the level of significance. 


\section{Results}

The radiographs of all 2,000 Chinese men (mean age 72.4 years, range 65-92 years) and 2,000 women (mean age 72.6 years, range $65-98$ years) were obtained. There was no difference in age between the two sexes $(P=0.25)$. None of these subjects' spines were diagnosed as having pathological fractures or diseases other than degenerative or osteoporotic changes. The radiographs of 1,994 male subjects (99.7 \%) and 1,996 female subjects $(99.8 \%$ ) were deemed to be of suitable film quality for analysis of spondylolisthesis. Prevalence of spondylolisthesis for men from L1 to L5 is listed in Table 1. In male subjects 380 out of 1,994 subjects $(19.1 \%)$ had at least one level of spondylolisthesis. Of these 380 subjects, $43(11.3 \%)$ had a slip at two or more levels, whereas the remaining 337 subjects $(88.7 \%$ ) had a slip at only a single level. Anterolisthesis was seen in $283(14.2 \%)$ of the 1,994 subjects, whereas retrolisthesis was seen in $85(4.3 \%)$ of the 1,994 subjects. Twelve $(0.6 \%)$ of the 1,994 subjects had concurrent anterolisthesis and retrolisthesis. A total of 427 out of 9,970 levels $(4.3 \%)$ assessed in male subjects had some degree of listhesis. Of these 427 vertebral levels, 414 (97.0\%) were Meyerding grade I spondylolisthesis and $12(2.8 \%)$ were Meyerding grade II spondylolisthesis.

Anterolisthesis was mainly grade I with a much smaller number of grade II and only one grade III (Table 1), whereas all retrolisthesis cases were grade I. Anterolisthesis was most commonly seen at the L4/5 level, whereas retrolisthesis was most commonly seen at the L3/4 level.

Prevalence of spondylolisthesis in women from L1 to L5 is listed in Table 2. In female subjects, 499 of the 1,996 subjects assessed $(25.0 \%)$ had at least one level of spondylolisthesis. Of these 499 subjects, 69 (13.8\%) had slips at two or more levels, whereas the remaining $430(86.2 \%)$ had a slip at a single level only. Of the 499 subjects with spondylolisthesis, anterolisthesis was seen in 459 subjects, whereas retrolisthesis was seen in $34(1.7 \%)$ subjects. Six $(0.3 \%)$ subjects had both anterolisthesis and retrolisthesis. A total of 575 out of 9,980 levels (5.8\%) assessed in female subjects had some degree of listhesis. Of these 575 vertebral levels, 545 (94.8 \%) were Meyerding grade I spondylolisthesis and 30 (5.2\%) Meyerding grade II spondylolisthesis. All retrolisthesis cases were grade 1 (Table 2). Similar to male subjects, in female subjects anterolisthesis was most commonly seen at the L4/L5 level, whereas retrolisthesis was most commonly seen at the L3/L4 level.

Spondylolisthesis characteristics for men and women are shown in Table 3. The ratio of male to female spondylolisthesis prevalence was 1:1.3, whereas that for anterolisthesis was 1:1.6. Women had a higher prevalence of spondylolisthesis than men $(P<0.001)$. Women also had the tendency towards a greater prevalence of grade II spondylolisthesis $(P=0.07)$. Men were more likely to have retrolisthesis $(P<0.001)$.

Age-specific prevalence of spondylolisthesis is shown in Table 4. Spondylolisthesis increased with age among both men $(P<0.0001$ for trend) and women $(P=0.014$ for trend).

Demographic and clinical data separated according to sex for subjects with and without spondylolisthesis are summarised in Table 5. For both men and women, advanced age, short height, higher BMI, higher spine and hip BMD, and clinical degenerative arthritis are associated with increased likelihood of spondylolisthesis. A lower PASE score was associated with increased likelihood of spondylolisthesis in men, whereas a higher body weight, angina and lower grip strength were associated with increased likelihood of spondylolisthesis in women. Spondylolisthesis was significantly associated with low back pain in men, whereas this association did not reach statistical significance in women. For both men and women, subjects with spondylolisthesis tended to have difficulty in bending 
forwards, but statistical significance was not reached. Factors including current smoking and alcohol consumption, COPD, history of fracture, corticosteroid intake, dietary calcium intake, diabetes, age at menopause, longest occupation involving physical labour, number of children and hormone replacement therapy were not associated with spondylolisthesis. Logistic regression analysis showed that advanced age and higher BMD at either the hip or spine were significant predictors of spondylolisthesis for both men and women. In addition, low back pain was a significant predictor of spondylolisthesis for men, whereas lower grip strength was a significant predictor of spondylolisthesis for women (Table 6).

\section{Discussion}

Despite the frequency of surgery performed for spondylolisthesis, the epidemiology of this condition remains unclear. One strength of this study is that we performed an investigation in both men and women from the same community-based population, and lateral lumbar spine radiographs were obtained in all subjects. The design of this study in Chinese subjects was the same as the Osteoporotic Fractures in Men (MrOS, USA) and Osteoporotic Fractures in Women (MsOS, USA) studies carried out on mainly Caucasian patients. Although the US studies on lumbar spondylolisthesis $[15,18]$ contained fewer subjects (295 men, 788 women), some insight into racial differences could be obtained by comparing these studies. In addition, prevalence of retrolisthesis was assessed in our cohort. There has been little previous research on the prevalence of retrolisthesis. However, retrolisthesis may be more strongly associated with low back pain and impaired lumbar function [15]. The present study found a prevalence of lumbar spondylolisthesis of $19.1 \%$ in elderly Chinese men and $25.0 \%$ in elderly Chinese women.

Compared with the MrOS (USA) study, our male cohort had a lower anterolisthesis prevalence (19.1\% versus $31 \%$ ) [18], more Meyerding grade II anterolisthesis (2.8 \% vs. 1 $\%)$ and slightly more subjects with more than one level of anterolisthesis (5.1\% vs. $4 \%)$ [18]. For women, in the MsOS (USA) study Vogt et al. used greater than $3 \mathrm{~mm}$ as the threshold of spondylolisthesis and measured at the lower lumbar level (L3 to S1) [15]. The anterolisthesis prevalence was $28.9 \%$ and that of retrolisthesis was $14.2 \%$. When the cutoff point is increased to $5 \mathrm{~mm}$, the prevalence decreases to $14.2 \%$ and $3.2 \%$ respectively [15]. Vogt et al.'s results have been considered to be similar to those of a population-based radiographic survey conducted in 1982 in the Netherlands [24]. Similar to our study, $73 \%$ of anterolisthesis in the MsOS (USA) study involved the L4-L5 level, and retrolisthesis involved primarily the L3-L4 or L5-S1 levels instead of L4/5 [15].

No similar studies in community-dwelling subjects have been performed in Asian populations. In one study of Japanese subjects with low back pain, $8.7 \%$ patients showed degenerative spondylolisthesis (defined as a slippage of $>3 \mathrm{~mm}$, including both anterolisthesis and retrolisthesis, but excluding spondylolytic spondylolisthesis [25]. Chen et al. reported that among Taiwanese taxi drivers over 45 years old with low back pain, the degenerative spondylolisthesis prevalence was $8.9 \%$ [13].

The aetiology of spondylolisthesis is multifactorial. Disc and facet joint degeneration are generally considered the most likely causes [26-28], although increased sagittal orientation of the facet joints, loss of paraspinal muscle bulk [29-32], increasing age [17, 26], strength of the iliolumbar ligaments, increased pelvic inclination or reclination, and increased L1-S1 angulation have all been considered to be potential causes to varying degrees in cadaver and radiological studies [18].

The present study confirmed previous reports that women are more susceptive to spondylolisthesis than men. The male to female ratio of 1:1.3 (or 1:1.6 for anterolisthesis) in 
this study is, however, considerably lower than in previous population-based studies on Caucasian subjects $[4,18,19]$. In the Danish study, Jacobsen et al. reported a male to female ratio of 1:6.4 [14]. Pregnancy [33], generalised joint laxity [34] and oophorectomy [35] are thought to be predisposing systemic factors in spondylolisthesis as well as a postmenopausal status [14]. Wang et al. recently demonstrated that elderly women had more severe disc narrowing than their male age-matched counterparts [36-38]. The higher severity of disc degeneration in elderly women may partially be responsible for the higher prevalence of degenerative spondylolisthesis in women than in men. Our results suggested that higher hip $\mathrm{BMD}$ and spine BMD are associated with higher prevalence of spondylolisthesis. We have previously suggested that higher BMD is associated with more severe disc degeneration [36, 38]. It is likely that higher BMD leads to more severe disc degeneration, and in turn leads to higher degenerative spondylolisthesis prevalence.

In our study low back pain was significantly associated with spondylolisthesis in men, whereas Jacobsen et al. found no relationship between low back pain and degenerative spondylolisthesis [14]. This study further confirmed that the prevalence of spondylolisthesis increased with age in both men and women. Unsurprisingly, a history of self-reported degenerative arthritis is associated with a higher prevalence of spondylolisthesis because these subjects are more likely to have symptomatic or severe degenerative arthritis. Greater physical activity and frequent exercise could result in a higher prevalence of degenerative spondylolisthesis owing to increased loads upon the lumbar spine $[3,13,23,39,40]$. However, no association between spondylolisthesis and occupational exposure with repeated daily lifting was observed in the Copenhagen cohort [14]. Our results suggested that longest occupation involving physical labour was not associated with higher prevalence of spondylolisthesis. On the other hand, PASE score was significantly higher in male subjects without spondylolisthesis than in those with spondylolisthesis, whereas grip strength was significantly greater in female subjects without spondylolisthesis than in those with spondylolisthesis. Physical activity and greater grip strength may play a protecting role via stronger muscles to maintain the stability of the spine.

Similar to previous reports [13,14], our study showed higher BMI in men and women, and greater weight in women with spondylolisthesis. Biomechanically both the compression force and the axial load on lumbar facet joints are increased as the body mass increases [41]. Our elderly male and female subjects with spondylolisthesis were all shorter; this is probably because subjects with spondylolisthesis are more likely to have narrowed disc spaces [15]. Lifestyles, such as alcohol consumption and cigarette smoking, can affect bone health [42]. Others suggested that diabetes and atherosclerotic disease may be associated with degenerative spondylolisthesis [43]. In our study angina is significantly associated with spondylolisthesis in women, whereas dietary calcium intake, diabetes, history of fracture, smoking, alcohol consumption, corticosteroid intake and COPD were not associated with spondylolisthesis in either men or women. Age at menopause, hormone replacement therapy and number of children born was not associated with spondylolisthesis in women.

A limitation of the current study is that only relatively healthy subjects with a high likelihood of surviving the study duration were enrolled. This could have led to a selection bias in favour of relatively healthy participants. However, this may be an inevitable feature of medium to long-term prospective cohort studies. The study questionnaire was designed with a view to determining risk factors for osteoporosis and was not specifically designed to characterise risk factors for spondylolisthesis. Therefore the variables associated with spondylolisthesis could not be explored in greater depth. As in previous studies [13, 15, 28], recumbent lateral radiographs were used to assess spondylolisthesis in this study. Vertebral displacement resulting from spondylolisthesis measured on radiographs could decrease as the subjects' posture changed from upright to supine position; therefore, recumbent lateral 
radiographs may underestimate the prevalence and the degree of spondylolisthesis. It has been suggested that a portion of spondylolisthetic lesions can only be seen by a standing lateral radiograph [19]. However, in one experiment, mean slip distances observed among 125 patients with spondylolysis were not significantly different on recumbent and standing radiographs, being $12.0 \mathrm{~mm}( \pm 8.8)$ on recumbent and $12.3 \mathrm{~mm}( \pm 8.6)$ respectively [44]. In this study it was not possible to differentiate degenerative spondylolisthesis from spondylolytic spondylolisthesis. However, in elderly subjects, spondylolytic spondylolisthesis made up only a very small portion of total spondylolistheses [45]. Additionally, because the frontal view of the lumbar spine was not obtained, disc and facet joint degeneration could not be fully assessed.

In conclusion, this large-scale study on community-based populations demonstrated that the prevalence of spondylolisthesis was $19.1 \%$ in elderly Chinese men and $25.0 \%$ in elderly Chinese women. Anterolisthesis was most commonly seen at the L4/L5 level, whereas retrolisthesis was most commonly seen at the L3/L4 level, both for men and women. The prevalence of spondylolisthesis in elderly Chinese is broadly similar to that of the MsOS (USA) study, but lower than the MrOS (USA) study. The overall male to female ratio in Chinese subjects was 1:1.3 which is lower than reports for Caucasian subjects. Women tended to have more Meyerding grade II spondylolisthesis than men. On the other hand, men were more likely to have retrolisthesis than women. Higher physical activity and grip strength may protect against the occurrence of spondylolisthesis, whereas higher hip BMD and higher spine BMD, and self-reported degenerative arthritis were associated with a higher prevalence of spondylolisthesis. Our study also reinforced some of the previous studies in which advanced age and higher BMI were associated with a higher prevalence of spondylolisthesis.

\section{Acknowledgments}

This study was funded by the National Institute of Health R01 Grant AR049439-01A1 and the Research Grants Council Earmarked Grant CUHK 4101/02M.

\section{References}

1. Wiltse LL, Newman PH, Macnab I. Classification of spondylolisis and spondylolisthesis. Clin Orthop Relat Res. 1976:23-294. [PubMed: 1277669]

2. Fredrickson BE, Baker D, McHolick WJ, et al. The natural history of spondylolysis and spondylolisthesis. J Bone Joint Surg Am. 1984; 66:699-707. [PubMed: 6373773]

3. Beutler WJ, Fredrickson BE, Murtland A, et al. The natural history of spondylolysis and spondylolisthesis: 45-year follow-up evaluation. Spine. 2003; 28:1027-1035. [PubMed: 12768144]

4. North American Spine Society. Diagnosis and treatment of degenerative lumbar spondylolisthesis. North American Spine Society: Burr Ridge; 2008. Clinical guidelines for multidisciplinary spine care.

5. Rosenberg NJ. Degenerative spondylolisthesis Predisposing factors. J Bone Joint Surg Am. 1975; 57:467-474. [PubMed: 1141255]

6. Moller H, Sundin A, Hedlund R. Symptoms, signs, and functional disability in adult spondylolisthesis. Spine. 2000; 25:683-690. [PubMed: 10752099]

7. Weinstein JN, Lurie JD, Olson PR, et al. United States' trends and regional variations in lumbar spine surgery: 1992-2003. Spine. 2006; 31:2707-2714. [PubMed: 17077740]

8. Deyo RA, Gray DT, Kreuter W, et al. United States trends in lumbar fusion surgery for degenerative conditions. Spine. 2005; 30:1441-1445. [PubMed: 15959375]

9. Fischgrund JS, Mackay M, Herkowits HN, et al. 1997 Volvo award winner in clinical studies Degenerative lumbar spondylolisthesis with spinal stenosis: a prospective, randomized study comparing decompressive laminectomy and arthrodesis with and without spinal instrumentation. Spine. 1997; 22:2807-2812. [PubMed: 9431616] 
10. Kornblum MB, Fischgrund JS, Herkowitz HN, et al. Degenerative lumbar spondylolisthesis with spinal stenosis: a prospective long-term study comparing fusion and pseudarthrosis. Spine. 2004; 29:726-733. [PubMed: 15087793]

11. Vibert BT, Sliva CD, Herkowitz HN. Treatment of instability and spondylolisthesis: surgical versus nonsurgical treatment. Clin Orthop Relat Res. 2006; 443:222-227. [PubMed: 16462445]

12. Weinstein JN, Lurie JD, Tosteson TD, et al. Surgical versus nonsurgical treatment for lumbar degenerative spondylolisthesis. N Engl J Med. 2007; 356:2257-2270. [PubMed: 17538085]

13. Chen JC, Chan WP, Katz JN, et al. Occupational and personal factors associated with acquired lumbar spondylolisthesis of urban taxi drivers. Occup Environ Med. 2004; 61:992-998. [PubMed: 15550605]

14. Jacobsen S, Sonne-Holm S, Rovsing H, et al. Degenerative lumbar spondylolisthesis: an epidemiological perspective: The Copenhagen Osteoarthritis Study. Spine. 2007; 32:120-125. [PubMed: 17202902]

15. Vogt MT, Rubin D, Valentin RS, et al. Lumbar olisthesis and lower back symptoms in elderly white women The study of osteoporotic fractures. Spine. 1998; 23:2640-2647. [PubMed: 9854764]

16. Kauppila LI, Eustace S, Kiel DP, et al. Degenerative displacement of lumbar vertebrae A 25-year follow-up study in Framingham. Spine. 1998; 23:1868-1873. [PubMed: 9762744]

17. Kalichman L, Kim DH, Li L, et al. Spondylolysis and spondylolisthesis: Prevalence and association with low back pain in the adult community-based population. Spine. 2009; 34:199205. [PubMed: 19139672]

18. Denard PJ, Holton KF, Miller J, et al. Lumbar spondylolisthesis among elderly men: prevalence, correlates and progression. Spine. 2010; 35:1072-1078. [PubMed: 20393398]

19. Fitzgerald J, Newman P. Degenerative spondylolisthesis. J Bone Joint Surg Br. 1976; 58:184-192. [PubMed: 932080]

20. Herkowitz H, Kurz L. Degenerative lumbar spondylolisthesis with spinal stenosis. J Bone Joint Surg Am. 1991; 73:802-808. [PubMed: 2071615]

21. Leung SS, Woo J, Ho S, Lam TH, Janus ED. Hong Kong adult dietary survey, 1995. Aust J Nutrit Diet. 1998; 55(Suppl 1):S11-S13.

22. Liu B, Woo J, Tang N, Ng K, Ip R, Au A. Assessment of total energy expenditure in a Chinese population by a physical activity questionnaire: examination of validity. Int J Food Sci Nutr. 2001; 52:269-282. [PubMed: 11400476]

23. Meyerding HW. Spondyloptosis. Surg Gynaecol Obstet. 1932; 54:371-377.

24. Valkenburg, HA.; Haanen, HCM. The epidemiology of low back pain. In: White, AA., editor. Proceedings from the American Association of Orthopaedic Surgeons Symposium on Low Back Pain. Rosemont: American Academy of Orthopaedic Surgeons; 1982. p. 9-22.

25. Iguchi T, Wakami T, Kurihara A, Kasahara K, Yoshiya S, Nishida K. Lumbar multilevel degenerative spondylolisthesis: radiological evaluation and factors related to anterolisthesis and retrolisthesis. J Spinal Disord Tech. 2002; 15:93-99. [PubMed: 11927816]

26. Herkowitz HN. Spine update. Degenerative lumbar spondylolisthesis. Spine. 1995; 20:1084-1090. [PubMed: 7631240]

27. Butler D, Trafimow JH, Andersson GBJ, et al. Discs degenerate before facets. Spine. 1990; 15:111-113. [PubMed: 2326704]

28. Rosenberg NJ. Degenerative spondylolisthesis. Predisposing factors. J Bone Joint Surg Am. 1975; 57:467-474. [PubMed: 1141255]

29. Grobler L, Robertson P, Novotny J. Etiology of spondylolisthesis. Assessment of the role played by of lumbar facet joint morphology. Spine. 1993; 18:80-91. [PubMed: 8434330]

30. Love T, Fagan A, Fraser R. Degenerative spondylolisthesis. Developmental or acquired? J Bone Joint Surg Br. 1999; 81:670-674. [PubMed: 10463743]

31. Matsunaga S, Sakou TMY. Natural history of degenerative spondylolisthesis. Pathogenesis and natural course of the slippage. Spine. 1990; 15:1204-1210. [PubMed: 2267617]

32. McAfee P, Yuan H. Computed tomography in spondylolisthesis. Clin Orthop Relat Res. 1982; 166:62-71. [PubMed: 7083687] 
33. Sanderson PL, Fraser RD. The influence of pregnancy on the development of degenerative spondylolisthesis. J Bone Joint Surg Br. 1996; 78:951-954. [PubMed: 8951013]

34. Porter RW, Hibbert C. Vertebral displacement in spondylolisthesis. Clin Biomech. 1989; 4:58-63.

35. Imada K, Matsui H, Tsuji H. Oophorectomy predisposes to degenerative spondylolisthesis. J Bone Joint Surg Br. 1995; 77:126-130. [PubMed: 7822369]

36. Wang YX, Griffith JF, Ma HT, Kwok AW, Leung JC, Yeung DK, Ahuja AT, Leung PC. Relationship between gender, bone mineral density, and disc degeneration in the lumbar spine: a study in elderly subjects using an eight-level MRI-based disc degeneration grading system. Osteoporos Int. 2011; 22:91-96. [PubMed: 20352410]

37. Wang YX, Griffith J. Effect of menopause on lumbar disk degeneration: potential etiology. Radiology. 2010; 257:318-320. [PubMed: 20959546]

38. Wang YX, Griffith JF, Zeng XJ, Deng M, Kwok AW, Leung JC, Ahuja AT, Kwok T, Leung P. Prevalence and sex difference of lumbar disc space narrowing in elderly Chinese men and women: osteoporotic fractures in men (Hong Kong) and osteoporotic fractures in women (Hong Kong) studies. Arthritis Rheum. 2013; 65:1004-1010. [PubMed: 23335175]

39. Newman P, Stone K. The etiology of spondylolisthesis. J Bone Joint Surg. 1963; 45-B:39-59.

40. Mariconda M, Galasso O, Imbimbo L, et al. Relationship between alterations of the lumbar spine, visualized with magnetic resonance imaging, and occupational variables. Eur Spine J. 2007; 16:255-266. [PubMed: 16835739]

41. Farfan HF, Osteria V, Lamy C. The mechanical etiology of spondylolysis and spondylolisthesis. Clin Orthop Related Res. 1976; 117:40-55.

42. Griffith JF, Kumta SM, Huang Y. Hard arteries, weak bones. Skeletal Radiol. 2011; 40:517-521. [PubMed: 21359689]

43. Farfan HF. The pathological anatomy of degenerative spondylolisthesis. A cadaver study. Spine. 1980; 5:412-418. [PubMed: 7455772]

44. Lowe RW, Hayes TD, Kaye J, et al. Standing roentgenograms in spondylolisthesis. Clin Orthop Relat Res. 1976; 10:80-84. [PubMed: 1277688]

45. Hu SS, Tribus CB, Diab M, Ghanayem AJ. Spondylolisthesis and spondylolysis. Instr Course Lect. 2008; 57:431-445. [PubMed: 18399601] 


\section{Key Points}

- $\quad$ The prevalence of spondylolisthesis is $19.1 \%$ in elderly Chinese men.

- The prevalence of spondylolisthesis is $25.0 \%$ in elderly Chinese women.

- Men are more likely to have retrolisthesis.

- Anterolisthesis is most commonly seen at the L4/L5 level.

- Retrolisthesis is most commonly seen at the L3/L4 level. 


\begin{tabular}{|c|c|c|c|c|c|c|c|c|}
\hline & 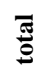 & $\stackrel{\infty}{\AA}$ & $\simeq$ & - & $\bar{m}$ & $\stackrel{0}{=}$ & $\stackrel{\Xi}{\forall}$ & $\hat{g}$ \\
\hline & $\sqrt[5]{3}$ & in & $r$ & - & 8 & $\stackrel{\infty}{\sim}$ & $\approx$ & $\bar{\infty}$ \\
\hline$\frac{\bar{d}}{\frac{\bar{d}}{\bar{d}}}$ & $\stackrel{\text { mo }}{\mathrm{g}}$ & $\overline{\vec{v}}$ & in & 0 & సิ & $=$ & సે & శે \\
\hline D. & $\stackrel{5}{3}$ & $\ddot{\imath}$ & 0 & 0 & $a$ & in & $\therefore$ & $\approx$ \\
\hline & తి & - & 0 & 0 & - & ते & in & in \\
\hline & $\stackrel{\mathrm{S}}{\mathrm{J}}$ & 0 & 0 & 0 & 0 & $N$ & $N$ & $N$ \\
\hline & & 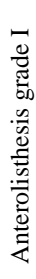 & 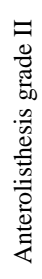 & 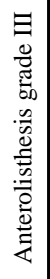 & 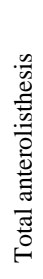 & 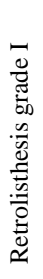 & 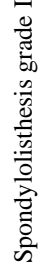 & 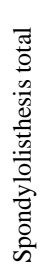 \\
\hline
\end{tabular}

Eur Radiol. Author manuscript; available in PMC 2015 February 01. 


\begin{tabular}{|c|c|c|c|c|c|c|c|}
\hline \multirow{6}{*}{ 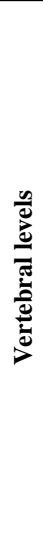 } & 氶 & \& & i & $\begin{array}{l}\text { in } \\
\text { ñ. }\end{array}$ & $g$ & 怼 & $\frac{n}{n}$ \\
\hline & $\sqrt[5]{3}$ & F & $\simeq$ & $\alpha$ & $r$ & $\begin{array}{l} \pm \\
\infty\end{array}$ & $\stackrel{8}{-}$ \\
\hline & $\stackrel{\text { fo }}{\mathrm{s}}$ & 志 & $\stackrel{2}{2}$ & 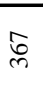 & in & 今ે & $\underset{m}{\stackrel{\sim}{m}}$ \\
\hline & $\stackrel{5}{3}$ & $\delta$ & - & 8 & $\bar{\sim}$ & 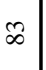 & $\underset{\infty}{ \pm}$ \\
\hline & తి & $m$ & & $m$ & $\cong$ & 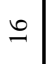 & $\stackrel{\circ}{\circ}$ \\
\hline & 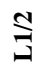 & & & & $m$ & $m$ & $m$ \\
\hline & & 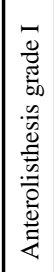 & 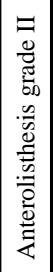 & 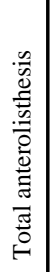 & 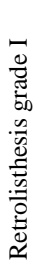 & 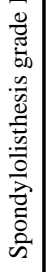 & 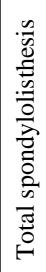 \\
\hline
\end{tabular}

Eur Radiol. Author manuscript; available in PMC 2015 February 01. 


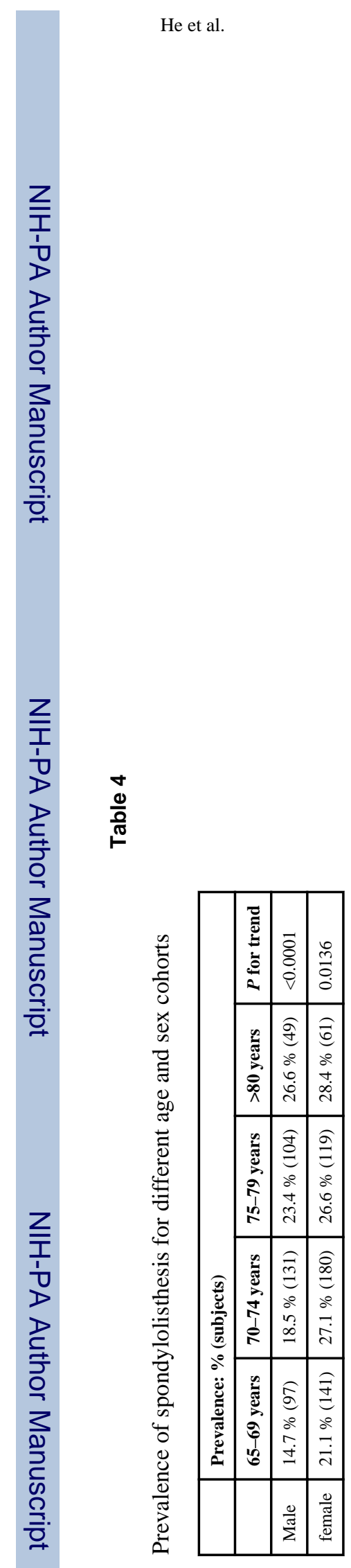

Eur Radiol. Author manuscript; available in PMC 2015 February 01. 
